\title{
Water-filling for full-duplex multiple-hop MIMO relay system
}

\author{
Pham Thanh Hiep ${ }^{1,2^{*}}$ and Ryuji Kohno ${ }^{1}$
}

\begin{abstract}
A water-filling (WF) method is one of the techniques to improve a performance of multiple-input multiple-output (MIMO) systems. However, the application of the WF method to multiple-hop MIMO relay systems (MMRSs) has not been discussed, especially in amplify-and-forward (AF) schemes. In this paper, the WF method for MMRSs with AF scheme is proposed and evaluated in both perfect and imperfect channel state information (CSI). The effect of imperfect CSI on optimization of transmit power at all transmitters and on co-channel interference is taken into account. Compared to the average transmit power, the end-to-end channel capacity of the WF method is higher in the perfect CSI; however, it is subject to the effect of imperfect CSI. Additionally, half-duplex and full-duplex transmission methods are also discussed in this paper. The end-to-end channel capacity of full duplex is higher and more robust than that of the half duplex due to a double of allocation time and a half of delay time.
\end{abstract}

Keywords: Water-filling method; Perfect and imperfect channel state information; Half duplex and full duplex; Multiple-hop MIMO relay systems; Amplify-and-forward scheme

\section{Introduction}

Multiple-input multiple-output (MIMO) relay systems have been discussed in several literatures [1-4]. According to transmission of MIMO schemes, a channel capacity of system was improved. Moreover, the allocation of transmit power for each antenna based on channel model, meaning water-filling (WF), also has been applied to improve the channel capacity of MIMO systems [5-8].

Additionally, in order to reduce the transmit power and/or improve the performance of MIMO system, a multiple-hop MIMO relay system (MMRS) has been analyzed [9-11]. In MMRSs, when the distance between the base station ( $\mathrm{Tx}$ ) and the final receiver ( $\mathrm{Rx})$ is fixed, the distance between the Tx to a relay (RS), a RS to a RS, a RS to the Rx called the distance between each transceiver, is shorten. Consequently, the signal-to-noise ratio (SNR) and the channel capacity are increased. However, according to the number of relays, the location, and the transmit power of each relay, the channel capacity of each hop is changed. In addition, the end-to-end channel capacity is

\footnotetext{
*Correspondence: phamthanhhiep@gmail.com

1 Yokohama National University, 79-5 Tokiwadai, Hodogaya, Yokohama 240-8501, Japan

${ }^{2}$ Le Quy Don Technical University, 236 Hoang Quoc Viet, Nghia Do, Cau Giay, Ha Noi 100000, Vietnam
}

restricted by the bottleneck node. Therefore, to obtain the upper bound of end-to-end channel capacity, the location of each relay node, meaning, the distance between each transceiver and the transmit power of each relay, should be optimized. We have analyzed the performance of MMRS with amplify-and-forward (AF) scheme [12,13] and decode-and-forward (DF) scheme [14]. The distance between each transceiver as well as the transmit power for each relay are optimized to obtain the upper bound of end-to-end channel capacity. However, the transmit power of each relay was divided equally to all antennas in the relay.

Since the DF relay decodes the received data before forwarding, it is known as a regenerative strategy. Furthermore, since each hop is independent of the other hops in the DF scheme MIMO relay system, the original WF method of a MIMO system can be directly applied. On the other hand, the AF relay amplifies the received data only; therefore it is known as a non-regenerative strategy. The computational simplicity in AF relay makes it a highly attractive and a strong candidate for the real-time application. Therefore, in this paper, we focus on the AF scheme MIMO relay system.

\section{是 Springer}

(c) 2014 Thanh Hiep and Kohno; licensee Springer. This is an Open Access article distributed under the terms of the Creative Commons Attribution License (http://creativecommons.org/licenses/by/2.0), which permits unrestricted use, distribution, and reproduction in any medium, provided the original work is properly credited. 
There are many literatures that focus on the AF scheme MIMO relay network $[9,10]$. In addition, the mutual information and the total mean square error criteria were selected as objective functions $[15,16]$. The diversity multiplexing tradeoff for MIMO relays also was investigated [17]. There are some works on the beamforming design for the special type of half-duplex AF scheme MIMO relay system [18]. However, in these literatures, the WF method was not applied.

In short, the WF method was proposed for the original MIMO system and the relay system which has only one relay. The WF method can be directly applied to DF scheme MMRSs. Moreover, the perfect channel state information (CSI) and the half duplex were assumed. In this paper, the WF method for AF scheme MMRSs is proposed and compared to the original WF of MIMO system. The half-duplex as well as the full-duplex transmission schemes are analyzed based on the end-to-end channel capacity. Moreover, the perfect and the imperfect CSI are taken into account. Three schemes of channel model are proposed, and the end-to-end channel capacity is analyzed based on the proposed scheme by the theoretical calculation method.

The rest of the paper is organized as follows. We introduce the concept of MMRSs in Section 2. The WF method for AF scheme MMRSs is proposed in Section 3, and the numerical evaluation is described in Section 4. Finally, Section 5 concludes the paper.

\section{AF scheme MMRSs}

\subsection{Description of MMRSs}

As shown in Figure 1, the MMRS that consists of a single transmitter, single receiver, and $m$ relays is considered. Let the Tx, the $\mathrm{Rx}$, and $R S_{i} \quad(i=1, \cdots, m)$ denote the transmitter, the receiver, and $i$ th relay, respectively. $M, N$, and $K_{i} \quad(i=1, \cdots, m)$ are the number of antennas at the $\mathrm{Tx}$, the $\mathrm{Rx}$, and each relay. However, in this paper, the

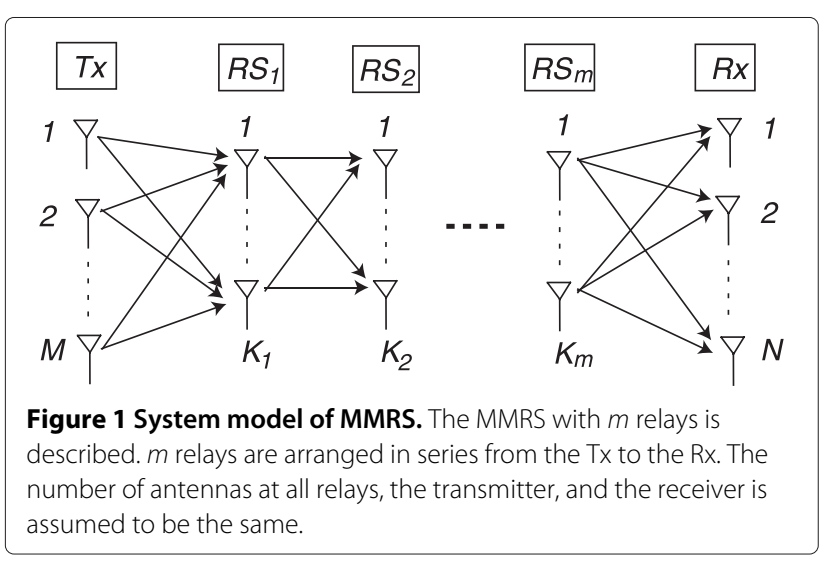

number of antennas of all relays is assumed to be the same as the number of antennas of the transmitter and the final receiver, $M=N=K_{i}(i=1, \cdots, m)$. Moreover, the total transmit power of all antennas in the $i$ th relay is denoted by $W_{i}$, for all $i=0, \cdots, m$. Since the AF method is applied to the channel model, the noise is also amplified and transmitted to the next relay when the signal is amplified at every relay. Mathematical notations used in this paper are as follows. $x$ and $X$ are scalar variables, $\mathbf{x}$ and $\mathbf{X}$ are vector variables or matrix variables, $\mathbf{X}^{\mathrm{H}}$ is conjugate transpose matrix of $\mathbf{X}$.

\subsection{Channel model}

In order to easily describe, the $\mathrm{Tx}$ and $\mathrm{Rx}$ are also be denoted as the $R S_{0}$ and $R S_{m+1}$, respectively. Since the path loss is taken into consideration, the channel matrix is a composite matrix, and we model as $\sqrt{l_{i i+1}} \mathbf{H}_{i i+1}, \quad i=0, \cdots, m$, of which $l_{i i+1}$ and $\mathbf{H}_{i i+1}$ represent the path loss and the $K_{i+1} \times K_{i}$ channel matrix between the $R S_{i}$ and the $R S_{i+1}$, respectively. $\mathbf{H}_{i i+1}$ is a matrix with independent and identical distribution (i.i.d.), zero mean, unit variance, circularly symmetric complex Gaussian entries.

Let $\mathbf{H}_{i}(i=1, \cdots, m+1)$ denote the channel matrix between the Tx and the $R S_{i}$ (as expressed in Figure 2). The other parameters of the system, i.e., transmit signal, received signal, noise vector, amplification factor, and total transmit power, are summarized in Table 1.

After $M \times 1$ normalized signal $\mathbf{S}$ is amplified by amplification factor $\Gamma_{\mathbf{0}}$, the signal $\mathbf{S}_{0}=\Gamma_{0} \mathbf{S}$ is transmitted to the $R S_{1}$. The signal $\mathbf{H}_{01} \mathbf{S}_{0}$ is received by the $R S_{1}$, and before being amplified with the amplification factor $\Gamma_{\mathbf{1}}$, the received signal is added by the $K_{1} \times 1$ noise matrix $\mathbf{n}_{1}$. Therefore, we can separate the received signal and the transmit signal of the $R S_{1}$ as

$$
\begin{aligned}
\mathbf{R}_{1} & =\mathbf{H}_{01} \mathbf{S}_{0}, \\
\mathbf{S}_{1} & =\boldsymbol{\Gamma}_{1}\left(\mathbf{R}_{1}+\mathbf{n}_{1}\right) .
\end{aligned}
$$

Similarly, when the signal $\mathbf{S}_{i-1}$ is transmitted from the $R S_{i-1}$, the received signal and the transmit signal of the $R S_{i}$ are expressed as

$$
\begin{aligned}
\mathbf{R}_{i} & =\mathbf{H}_{i-1 i} \mathbf{S}_{i-1}, \\
\mathbf{S}_{i} & =\boldsymbol{\Gamma}_{i}\left(\mathbf{R}_{i}+\mathbf{n}_{i}\right) .
\end{aligned}
$$

The amplification factor of the $R S_{i}$ is expressed as

$$
\begin{aligned}
\boldsymbol{\Gamma}_{0} & =\sqrt{\mathbf{P}_{0}} \\
\boldsymbol{\Gamma}_{0} & =\sqrt{\frac{\mathbf{P}_{i}}{\left|\mathbf{R}_{i}\right|^{2}}}, \quad i=1, \cdots, m,
\end{aligned}
$$

where $\mathbf{P}_{i}=\operatorname{diag}\left(p_{i 1}, p_{i 2}, \cdots, p_{i K_{i}}\right)$ is the transmit power matrix and assumed to be subject to a constraint $\operatorname{Tr}\left(\mathbf{P}_{i}\right)=$ $W_{i}$. The $\operatorname{Tr}(\cdot)$ and $p_{i j}$ denote the trace and transmit power 


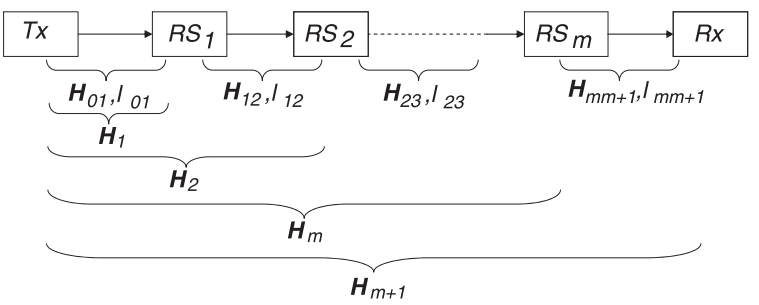

Figure $\mathbf{2}$ Channel model of MMRS. The channel model of each hop, the channel model from the Tx to each relay, and the path loss between each transceiver are described.

of the $j$ th antenna of the $R S_{i}$, respectively. Finally, the received signal at the $\mathrm{Rx}$ can be expressed as

$$
\mathbf{S}_{m+1}=\sqrt{\frac{l_{m m+1} \mathbf{P}_{m} \cdots l_{01} \mathbf{P}_{0}}{\left|\mathbf{R}_{m}\right|^{2} \cdots\left|\mathbf{R}_{1}\right|^{2}}} \mathbf{H}_{m m+1} \cdots \mathbf{H}_{12} \mathbf{H}_{01} \mathbf{S}_{0}+\mathbf{n}
$$

Here, the $\mathbf{n}$ denotes the noise vector of the system. Therefore, the system channel matrix is written by

$$
\mathbf{H}_{m+1}=\mathbf{H}_{m m+1} \cdots \mathbf{H}_{12} \mathbf{H}_{01} \text {. }
$$

According to using the system channel matrix $\mathbf{H}_{m+1}$, the MMRS can be analyzed as same as a conventional MIMO system.

\subsection{End-to-end channel capacity}

The noise vector of the system when $m$ relays intervened becomes

$$
\begin{aligned}
\mathbf{n}= & \sqrt{\frac{l_{m m+1} \mathbf{P}_{m} \cdots l_{12} \mathbf{P}_{1}}{\left|\mathbf{R}_{m}\right|^{2} \cdots\left|\mathbf{R}_{1}\right|^{2}}} \mathbf{H}_{m m+1} \cdots \mathbf{H}_{12} \mathbf{n}_{1} \\
& +\sqrt{\frac{l_{m m+1} \mathbf{P}_{m} \cdots l_{23} \mathbf{P}_{2}}{\left|\mathbf{R}_{m}\right|^{2} \cdots\left|\mathbf{R}_{1}\right|^{2}}} \sqrt{\left|\mathbf{R}_{1}\right|^{2}} \mathbf{H}_{m m+1} \cdots \mathbf{H}_{23} \mathbf{n}_{2} \\
& +\cdots \\
& +\sqrt{\frac{l_{m m+1} \mathbf{P}_{m}}{\left|\mathbf{R}_{m}\right|^{2} \cdots\left|\mathbf{R}_{1}\right|^{2}}} \sqrt{\left|\mathbf{R}_{m-1}\right|^{2} \cdots\left|\mathbf{R}_{1}\right|^{2}} \mathbf{H}_{m m+1} \mathbf{n}_{m} \\
& +\mathbf{n}_{m+1} .
\end{aligned}
$$

However, at first, the CSI is assumed to be perfect; the imperfect CSI is considered in the following section.

Table 1 System parameters of each transceiver

\begin{tabular}{lcclcc}
\hline & Tx & $\boldsymbol{R S}_{\mathbf{1}}$ & $\cdots$ & $\boldsymbol{R} \boldsymbol{S}_{\boldsymbol{m}}$ & $\mathbf{R x}$ \\
\hline Transmit signal & $\mathbf{S}_{0}$ & $\mathbf{S}_{1}$ & $\cdots$ & $\mathbf{S}_{m}$ & \\
Received signal & & $\mathbf{R}_{1}$ & $\ldots$ & $\mathbf{R}_{m}$ & $\mathbf{R}_{m+1}$ \\
Noise vector & & $\mathbf{n}_{1}$ & $\ldots$ & $\mathbf{n}_{m}$ & $\mathbf{n}_{m+1}$ \\
Amplification factor & $\boldsymbol{\Gamma}_{0}$ & $\boldsymbol{\Gamma}_{1}$ & $\cdots$ & $\boldsymbol{\Gamma}_{m}$ & \\
Total transmit power & $E_{0}$ & $E_{1}$ & $\cdots$ & $E_{m}$ & \\
\hline
\end{tabular}

The channel matrix $\mathbf{H}_{01}$ can be expressed as follows in a singular-value decomposition (SVD) $[19,20]$.

$$
\mathbf{H}_{01}=\mathbf{E}_{01_{r}} \mathbf{D}_{01} \mathbf{E}_{01_{t}}^{\mathrm{H}}=\sum_{i=1}^{M} \sqrt{\lambda_{i}} e_{01_{r, i}} e_{01_{t, i}}^{\mathrm{H}}
$$

here,

$$
\begin{aligned}
& \mathbf{D}_{01}=\operatorname{diag}\left[\sqrt{\lambda_{01_{1}}} \sqrt{\lambda_{01_{2}}} \cdots \sqrt{\lambda_{01_{M}}}\right],
\end{aligned}
$$

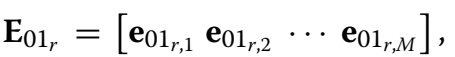

$$
\begin{aligned}
& \mathbf{E}_{01_{t}}=\left[\begin{array}{llll}
\mathbf{e}_{01_{t, 1}} & \mathbf{e}_{01_{t, 2}} \cdots \mathbf{e}_{01_{t, M}}
\end{array}\right] \text {. }
\end{aligned}
$$

$\lambda_{i}(i=1,2, \cdots, M)$ is the $i$ th eigenvalue of the correlation matrix $\mathbf{H}_{01} \mathbf{H}_{01}^{\mathrm{H}}$ (or $\mathbf{H}_{01}^{\mathrm{H}} \mathbf{H}_{01}$ ), $\mathbf{e}_{01_{r, i}}$ is the eigenvector belonging to the eigenvalue $\lambda_{i}$ of correlation matrix $\mathbf{H}_{01} \mathbf{H}_{01}^{\mathrm{H}}$, and $\mathbf{e}_{01_{t, i}}$ is the eigenvector belonging to the eigenvalue $\lambda_{i}$ of correlation matrix $\mathbf{H}_{01}^{\mathrm{H}} \mathbf{H}_{01}$. The SVD is applied similarly to the other channel matrices.

The channel matrix and the noise vector are assumed to be uncorrelated, and the covariance of noise vector in all relays is denoted by $\sigma^{2}$. Therefore, the correlation of noise vector of system is represented as follows.

$$
\begin{aligned}
& \mathbf{n n}^{\mathrm{H}} \\
& =\frac{\sum_{i=0}^{m} \prod_{j \neq i}^{m} l_{j j+1} \mathbf{P}_{j} \mathbf{D}_{j j+1}^{2} \sigma^{2}+\sigma^{4} f\left(l_{i i+1} \mathbf{P}_{i} \mathbf{D}_{i i+1}^{2}, \sigma^{2}\right)}{\left|\mathbf{R}_{m}\right|^{2} \cdots\left|\mathbf{R}_{1}\right|^{2}}
\end{aligned}
$$

here, $f\left(l_{i i+1} \mathbf{P}_{i} \mathbf{D}_{i i+1}^{2}, \sigma^{2}\right)$ denotes a polynomial of $l_{i i+1} \mathbf{P}_{i} \mathbf{D}_{i i+1}^{2}$ and $\sigma^{2}$. Since $\sigma^{2} \leq 1$ and the covariance of noise vector is smaller than the received power, the component containing $\sigma^{4}$ can be ignored. Thus, (9) can be changed as

$$
\mathbf{n n}^{\mathrm{H}}=\frac{\sum_{i=0}^{m} \prod_{j \neq i}^{m} l_{j j+1} \mathbf{P}_{j} \mathbf{D}_{j j+1}^{2} \sigma^{2}}{\left|\mathbf{R}_{m}\right|^{2} \cdots\left|\mathbf{R}_{1}\right|^{2}}
$$


Consequently, the end-to-end channel capacity of AF scheme MMRS is expressed as

$$
\begin{aligned}
C & =\log _{2}\left(\operatorname{det}\left(\frac{\mathbf{S}_{m+1} \mathbf{S}_{m+1}^{\mathrm{H}}}{\mathbf{n}_{m+1} \mathbf{n}_{m+1}^{\mathrm{H}}}\right)\right) \\
& =\log _{2}\left(\operatorname{det}\left(\mathbf{I}_{M}+\frac{\prod_{i=0}^{m} l_{j j+1} \mathbf{P}_{j} \mathbf{D}_{j j+1}^{2}}{\sigma^{2} \sum_{i=0}^{m} \prod_{j \neq i}^{m} l_{j j+1} \mathbf{P}_{j} \mathbf{D}_{j j+1}^{2}}\right)\right) \\
& =\log _{2}\left(\operatorname{det}\left(\mathbf{I}_{M}+\frac{1}{\sigma^{2} \sum_{i=0}^{m} \frac{1}{l_{i i+1} \mathbf{P}_{i} \mathbf{D}_{i i+1}^{2}}}\right)\right)
\end{aligned}
$$

here, $\mathbf{I}_{M}$ denotes the $M \times M$ unit matrix.

Let

$$
f(\lambda)=\sigma^{2} \sum_{i=0}^{m} \frac{1}{l_{i i+1} \mathbf{P}_{i} \mathbf{D}_{i i+1}^{2}} .
$$

Thus, the end-to-end channel capacity of system is inversely proportional to $f(\lambda)$. Therefore, $f(\lambda)$ can be considered instead of the end-to-end channel capacity. In order to maximize the end-to-end channel capacity, the $f(\lambda)$ has to be minimized. The $f(\lambda)$ is a function of transmit power and path loss, meaning, the distance between each transceiver, the variance of noise vector, and the matrix of eigenvalue of channel model. The optimization of distance and transmit power was described in [12,13]; however, the transmit power of each antenna in the same relay is assumed to be equal. In order to improve the performance of a system, the WF method for the AF scheme MMRS is proposed. The $f(\lambda)$ can be changed as

$$
f(\lambda)=\sum_{i=1}^{m+1} \frac{1}{\mathbf{S N R}_{i-1 i} \mathbf{D}_{i-1 i}^{2}} ;
$$

here, $\mathbf{S N R}_{i-1 i}=\frac{l_{i-1 i} \mathbf{P}_{i-1}}{\sigma^{2}}=\operatorname{diag}\left(\gamma_{i-1 i_{1}}, \gamma_{i-1 i_{2}}, \cdots\right.$, $\left.\gamma_{i-1 i_{M}}\right)$ represents the SNR of all paths of hop between the $R S_{i-1}$ and the $R S_{i}$ regardless the other relays. Since the transmit power and the distance are fixed after optimization as optimization method in $[12,13]$ and notice that $\operatorname{Tr}\left(\mathbf{P}_{i}\right)=W_{i}$, trace of $\mathbf{S N R}_{i-1 i}$ is fixed as $\frac{l_{i-1 i} W_{i-1}}{\sigma^{2}}=\operatorname{snr}_{i}$. $\mathrm{The} \mathrm{snr}_{i}$ is the ratio of received signal power to noise power where the signal is transmitted and received by one antenna.

\section{WF method for AF scheme MMRS}

\subsection{WF method for AF scheme MMRS with perfect CSI}

The WF method can be applied to the AF scheme MMRS by three schemes. Scheme 1: the WF method is applied to each hop in MMRS. Scheme 2: the WF method is applied to each process. Scheme 3: the WF method is applied to all channel models of MMRS. The detail of each scheme is explained as follows.

Scheme 1: the WF method for each hop of MMRS: In this scheme, the channel model of each hop is used to optimize the transmit power of transmitter. The hop between the $R S_{i-1}$ and the $R S_{i}$ is considered and regardless of the other relays. As described in $[7,8]$, in the system with only one transmitter and one receiver, the channel capacity is described as follows.

$$
\begin{aligned}
C_{i-1 i} & =\log _{2} \prod_{k=1}^{M}\left(1+\lambda_{i-1 i_{k}} \gamma_{i-1 i_{k}}\right), \\
& =\sum_{k=1}^{M} \log _{2} \lambda_{i-1 i_{k}}+\sum_{k=1}^{M} \log _{2}\left(\frac{1}{\lambda_{i-1 i_{k}}}+\gamma_{i-1 i_{k}}\right) .
\end{aligned}
$$

The channel capacity maximization problem is now changed to the choice of the maximal number of real, non-negative values $\gamma_{i-1 i_{k}}$ subject to the power constraint $\sum_{k=1}^{M} \gamma_{i-1 i_{k}}=\operatorname{snr}_{i}$.

The well-known approach to solving constrained optimization problems is the method of Lagrange multipliers. Accordingly, we need to maximize

$g(\gamma)=\sum_{k=1}^{M} \log _{2}\left(\frac{1}{\lambda_{i-1 i_{k}}}+\gamma_{i-1 i_{k}}\right)+\alpha\left(\sum_{k=1}^{M} \gamma_{i-1 i_{k}}-\mathrm{snr}_{i}\right)$,

where $\alpha$ is the Lagrange multiplier. The partial differentiation with respect to $\gamma_{i-1 i_{k}}$ leads to

$$
\frac{\partial g(\gamma)}{\partial \gamma_{i-1 i_{k}}}=\frac{\lambda_{i-1 i_{k}}}{1+\lambda_{i-1 i_{k}} \gamma_{i-1 i_{k}}}+\alpha \text {. }
$$

The optimized $\gamma_{i-1 i_{k}}$ can be obtained when $\frac{\partial g(\gamma)}{\partial \gamma_{i-1 i_{k}}}=0$. Hence,

$$
\gamma_{i-1 i_{k}}=-\frac{1}{\alpha}-\frac{1}{\lambda_{i-1 i_{k}}} .
$$

The Lagrange multiplier is relabeled as $\beta=-\frac{1}{\alpha}$ to simplify the notation in later formulas. This problem really has two constraints: the power constraint and being nonnegative $\gamma_{i-1 i_{k}}$. The latter constraint can be described as

$$
\begin{aligned}
\gamma_{i-1 i_{k}} & =\max \left\{0, \beta-\frac{1}{\lambda_{i-1 i_{k}}}\right\}, \\
& \equiv\left[\beta-\frac{1}{\lambda_{i-1 i_{k}}}\right]^{+} .
\end{aligned}
$$

The former constraint can be described as

$$
\begin{aligned}
\operatorname{snr}_{i} & =\sum_{k=1}^{M}\left[\beta-\frac{1}{\lambda_{i-1 i_{k}}}\right]^{+}, \\
& =\sum_{k=1}^{q}\left[\beta-\frac{1}{\lambda_{i-1 i_{k}}}\right]^{+}, \\
& =q \beta-\sum_{k=1}^{q} \frac{1}{\lambda_{i-1 i_{k}}} .
\end{aligned}
$$


Thus, the Lagrange multiplier and the optimal SNR, meaning, the optimal transmit power of each path responding to eigenvalues, are obtained.

$$
\begin{aligned}
\beta & =\frac{1}{q}\left(\sum_{k=1}^{q} \frac{1}{\lambda_{i-1 i_{k}}}+\mathrm{snr}_{i}\right), \\
\gamma_{i-1 i_{k}} & =\frac{1}{q}\left(\sum_{k=1}^{q} \frac{1}{\lambda_{i-1 i_{k}}}+\mathrm{snr}_{i}\right)-\frac{1}{\lambda_{i-1 i_{k}}} .
\end{aligned}
$$

A computational procedure for determining the optimal value of $q$ is to compute for $q=M, M-1, \cdots$ the value of $\gamma_{i-1 i_{k}}$ until this quantity is greater than zero for all $k$ from 1 to $q$. By substituting the optimal $\lambda_{i-1 i_{k}}$ to (13) and (11), the end-to-end channel capacity can be obtained.

Similar to scheme 1, in schemes 2 and 3, the transmit power of transmitter also can be optimized based on multiplication of channel models. In scheme 2: the WF method for each process, the transmit power of the transmitter is optimized based on multiplication of channel models from the Tx to each receiver. It means that the WF of hop between the $R S_{i-1}$ and the $R S_{i}$ is analyzed based on the channel model $\mathbf{H}_{i}=\mathbf{H}_{i-1 i} \cdots \mathbf{H}_{01}$ (refer to Figure 2). Let $\mathbf{D}_{i}$ denote the eigenvalue matrix of $\mathbf{H}_{i}$ in SVD; thus,

$$
\mathbf{D}_{i}=\mathbf{D}_{i-1 i} \cdots \mathbf{D}_{01}
$$

and the $\mathbf{S N R}_{i-1 i}$ is optimized based on $\mathbf{D}_{i}$ as mentioned above.

In scheme 3: the WF method for all channel models, the transmit power is optimized based on channel matrix of system, $\mathbf{H}_{m+1}$ in (5). The eigenvalue matrix of $\mathbf{H}_{m+1}$ is denoted by $\mathbf{D}_{m+1}$ that is represented as follows.

$$
\mathbf{D}_{m+1}=\mathbf{D}_{m m+1} \cdots \mathbf{D}_{01} \text {. }
$$

The WF method of all hops is dependent on $\mathbf{D}_{m+1}$; it means that the WF method of all hops is the same.

Up to now, the perfect CSI at both the transmitter and the receiver has been assumed, and the WF method for MMRS has been analyzed based on the proposed scheme. However, in actuality, the perfect CSI assumption is not always practical due to channel estimation errors, feedback delay, and noise. Compared to channel estimation errors, the CSI imperfection introduced by feedback channel delay is sometimes more significant and inevitable.

\subsection{WF method for AF scheme MMRS with imperfect CSI}

Similar to the previous section, the channel model between the $R S_{i-1}$ and the $R S_{i}$ is considered. The channel time variation is described by the first-order Markov process as follows.

$$
\mathbf{H}_{i-1 i}(t)=\rho \mathbf{H}_{i-1 i}(t-\tau)+\delta \overline{\mathbf{H}}_{i-1 i}(t),
$$

where $\tau$ denotes the total delay caused by signal processing, feedback, and other system delays; $\rho=J_{0}\left(2 \pi f_{D} \tau\right)$ is the time correlation coefficient; $J_{0}(\cdot)$ is the zero-th order
Bessel function of the first kind; and $f_{D}$ denotes the maximum Doppler frequency shift. $\delta$ denotes $\sqrt{1-\rho^{2}}$. The innovation term $\overline{\mathbf{H}}_{i-1 i}$ also has i.i.d entries, zero mean, unit variance, and circularly symmetric complex Gaussian entries. For notation brevity, we drop off the time index

$$
\mathbf{H}_{i-1 i}=\rho \hat{\mathbf{H}}_{i-1 i}+\delta \overline{\mathbf{H}}_{i-1 i},
$$

where $\hat{\mathbf{H}}_{i-1 i}$ denotes the outdated channel matrix while $\overline{\mathbf{H}}_{i-1 i}$ is the true one. Notice that there are two ways the outdated CSI affects the system. One is the effect on the separation of subchannel between each transceiver; the other one is the effect on optimization of transmit power of all transmitters. The latter one depends on the scheme of the WF method. The former one depends on the CSI between each transceiver regardless of other CSIs, and it is the same as the CSI of scheme 1 of the latter one. The CSI of the latter one is explained as follows. Notice that the full duplex is assumed, all terminals transmit and receive in the same time. The delay of half duplex is a double of full duplex.

For scheme 1: we assume that transmitters add the CSI symbol in data packets and transmit to receivers; hence, the receiver knows both $\overline{\mathbf{H}}_{i}$ and $\hat{\mathbf{H}}_{i}$. After receiving the CSI symbol, the receiver feedbacks the CSI to the transmitter immediately. However, the transmitter receives the CSI after a delay. Therefore, the CSI that is used at the transmitter is the outdated CSI. It means that the transmitter only knows the outdated channel model, $\hat{\mathbf{H}}_{i}$, while the receiver knows both $\overline{\mathbf{H}}_{i}$ and $\hat{\mathbf{H}}_{i}$. The delay at each hop is assumed to be the same as $\tau$. Therefore, in scheme 1 , the delay of CSI at each transmitter is the same as $\tau$.

For scheme 2: the CSI symbol that was added at the Tx is assumed to be forwarded at all relays; therefore, all relays and the Rx can know the CSI from the Tx to itself. However, when a relay receives the CSI symbol, the CSI of the previous hop becomes the outdated. Notice that the delay of forward link (from the Tx to the Rx) and backward link (from the Rx to the Tx) was assumed to be the same as $\tau$. The $R S_{i-1}$ starts to optimize the transmit power after receiving the feedback CSI from the $R S_{i}$; therefore, although the $R S_{i-1}$ receives the CSI of hop between the Tx and the $R S_{1}$ after the delay of $(i-2) \tau$, however, it should wait to receive the feedback from the $R S_{i}$; hence the $R S_{i-1}$ starts to optimize the transmit power when the delay is $i \tau$. Similarly, the delay of channel model between the $R S_{j-1}$ and the $R S_{j}$ becomes $(i-j+1) \tau$. As a result, the delay of CSI of $\mathbf{H}_{i-1 i}, \cdots, \mathbf{H}_{01}$ is $1 \tau, \cdots, i \tau$, respectively.

For scheme 3: we assumed that a relay $\left(R S_{i}\right)$ not only feedbacks the CSI to the previous relay $\left(R S_{i-1}\right)$ but also forwards the CSI received from the next relay $\left(R S_{i+1}\right)$ to the previous relay $\left(R S_{i-1}\right)$. The process is repeated until the Tx receives the CSI from the Rx. All transmitters optimize their transmit power every time the CSI is updated; however, as analyzed in the previous section, at all relays, 
the transmit power is optimized based on the same multiplication of channel models. Therefore, the delay of CSI is calculated based on the same channel models. When the Tx transmits a signal, the delay of CSI of $\mathbf{H}_{01}, \cdots, \mathbf{H}_{m m+1}$ is $\tau, \cdots,(m+1) \tau$, respectively. Compared to the transmission of the $\mathrm{Tx}$, the $R S_{i}$ transmits a signal after the delay of $i \tau$; therefore, the delay of CSI of $\mathbf{H}_{01}, \cdots, \mathbf{H}_{m m+1}$ is $(i+1) \tau, \cdots,(i+m+1) \tau$, respectively.

The influence of outdated CSI on the separation of subchannel of MIMO scheme is explained as follows. In a SVD-based MIMO system, the $R S_{i-1}$ steers the modulated signal vector, $\mathbf{S}_{i-1}$, within the eigenspace spanned by the right singular vectors contained in $\hat{\mathbf{E}}_{i-1 i_{t}}$.

$$
\mathbf{S}_{i-1_{t}}=\hat{\mathbf{E}}_{i-1 i_{t}} \mathbf{S}_{i-1} \text {. }
$$

The $R S_{i}$ receives the signal with noise vector as the same in Section 2.

$$
\mathbf{R}_{i_{r}}=\mathbf{H}_{i-1 i} \mathbf{S}_{i-1_{t}}+\mathbf{n}_{i}
$$

and the preprocesses $\mathbf{R}_{i_{r}}$ using $\hat{\mathbf{E}}_{i-1 i_{r}}$

$$
\begin{aligned}
\mathbf{R}_{i}= & \left(\hat{\mathbf{E}}_{i-1 i_{r}}\right)^{\mathrm{H}} \mathbf{R}_{i_{r}}, \\
= & \left(\hat{\mathbf{E}}_{i-1 i_{r}}\right)^{\mathrm{H}} \mathbf{H}_{i-1 i} \hat{\mathbf{E}}_{i-1 i_{t}} \mathbf{S}_{i-1}+\left(\hat{\mathbf{E}}_{i-1 i_{r}}\right)^{\mathrm{H}} \mathbf{n}_{i}, \\
= & \rho \hat{\mathbf{D}}_{i-1 i} \mathbf{S}_{i-1}+\delta\left(\hat{\mathbf{E}}_{i-1 i_{r}}\right)^{\mathrm{H}} \overline{\mathbf{H}}_{i-1 i} \hat{\mathbf{E}}_{i-1 i_{t}} \mathbf{S}_{i-1} \\
& +\left(\hat{\mathbf{E}}_{i-1 i_{r}}\right)^{\mathrm{H}} \mathbf{n}_{i+1} .
\end{aligned}
$$

The $p$ th component of vector $\mathbf{x}$ and the $p$ th row of matrix $\mathbf{X}$ are denoted by $\mathbf{x}[p]$ and $\mathbf{X}[p]$, respectively. The component-wise form is described as follows.

$$
\begin{aligned}
\mathbf{R}_{i}[p]= & \left(\rho \hat{\mathbf{D}}_{i-1 i}[p]+\delta\left(\hat{\mathbf{E}}_{i-1 i_{r}}\right)^{\mathrm{H}} \overline{\mathbf{H}}_{i-1 i} \hat{\mathbf{E}}_{i-1 i_{t}}[p]\right) \mathbf{S}_{i-1} \\
& +\delta \sum_{t \neq p}\left(\hat{\mathbf{E}}_{i-1 i_{r}}\right)^{\mathrm{H}} \overline{\mathbf{H}}_{i-1 i} \hat{\mathbf{E}}_{i-1 i_{t}}[t] \mathbf{S}_{i-1} \\
& +\left(\hat{\mathbf{E}}_{i-1 i_{r}}\right)^{\mathrm{H}} \mathbf{n}_{i+1}[p]
\end{aligned}
$$

where the received signal consists of three components: the information carrying term, the interference term, and the noise term. For each component-wise, there are $M-1$ interference components that are multiplied by $\delta$. The correlation matrix is approximate to the unit matrix. Therefore, (13) is changed as

$$
\begin{aligned}
& f(\lambda) \\
& =\frac{\sigma^{2} \sum_{i=0}^{m} \prod_{j \neq i}^{m} l_{j j+1} \mathbf{P}_{j} \mathbf{D}_{j j+1}^{2}+\delta(M-1) \prod_{i=0}^{m} l_{i i+1} \mathbf{P}_{i} \mathbf{D}_{i i+1}^{2}}{\prod_{i=0}^{m} l_{i i+1} \mathbf{P}_{i} \mathbf{D}_{i i+1}^{2}}, \\
& =\sum_{i=1}^{m+1} \frac{1}{\mathbf{S N R}_{i-1 i} \mathbf{D}_{i-1 i}^{2}}+\delta(M-1) .
\end{aligned}
$$

Compared to the system has the perfect CSI at both the transmitter and the receiver (13), in the function $f(\lambda)$ of the system that has the outdated CSI at the transmitter (29), the co-channel interference by the outdated CSI is added.

\section{Numerical evaluation}

\subsection{Effects of WF method}

The transmit power and the distance have been optimized based on different numbers of relays in $[12,13]$; thus, in this paper, the number of relays is fixed as 4 , and the SNR of all relays is assumed to be the same. The number of antennas of all relays, the Tx and the $\mathrm{Rx}$ is set as 6 . Since the performance of the system should be discussed in both the low-SNR region and the high-SNR region, the $\mathrm{snr}_{i}, i=$ $1, \cdots, m+1$ is changed from 0 to $30 \mathrm{~dB}$. Figure 3 shows the end-to-end channel capacity of the proposed WF method. In order to evaluate the proposed WF method, the result of the average transmit power, meaning, the transmit power of all antennas in one relay is equal, also is represented. The end-to-end channel capacity of average transmit power and three WF methods is compared. The end-to-end channel capacity of scheme 3 is the highest and that of scheme 2 is higher than that of scheme 1 . It can be explained that the transmit power of all transmitters in scheme 3 is the most suitable to channel model, and the transmit power of all transmitters in scheme 2 is more suitable to channel model than that of scheme 1. Similarly, the system with WF methods has more diversity than the system with average transmit power; therefore, the endto-end channel capacity of WF methods is higher than that of the average transmit power. Although the number of antennas in the full-duplex system is a half of the number of antennas in the half-duplex system, the allocation time of the full-duplex system is a double of allocation time of the half-duplex system. Hence, compared to the half duplex, the end-to-end channel capacity of full duplex is higher.

\subsection{System with outdated CSI}

As explained in Section 3.2, there are two ways the outdated CSI affects the system. One is the effect on optimizing the transmit power of all transmitters; the other one is the effect on co-channel interference. At first, the end-to-end channel capacity is calculated when the term $f_{D} \tau$ is changed; the $\operatorname{snr}_{i}, i=1, \cdots, m+1$ is fixed as 5 $\mathrm{dB}$ (in the low-SNR region) and $20 \mathrm{~dB}$ (in the high-SNR region). The full-duplex and the half-duplex systems are also compared. Figures 4 and 5 show the end-to-end channel capacity of full duplex and half duplex when the $\mathrm{snr}_{i}$ is 5 and $20 \mathrm{~dB}$, respectively. The end-to-end channel capacity decreases when the term $f_{D} \tau$ increases; however, the 


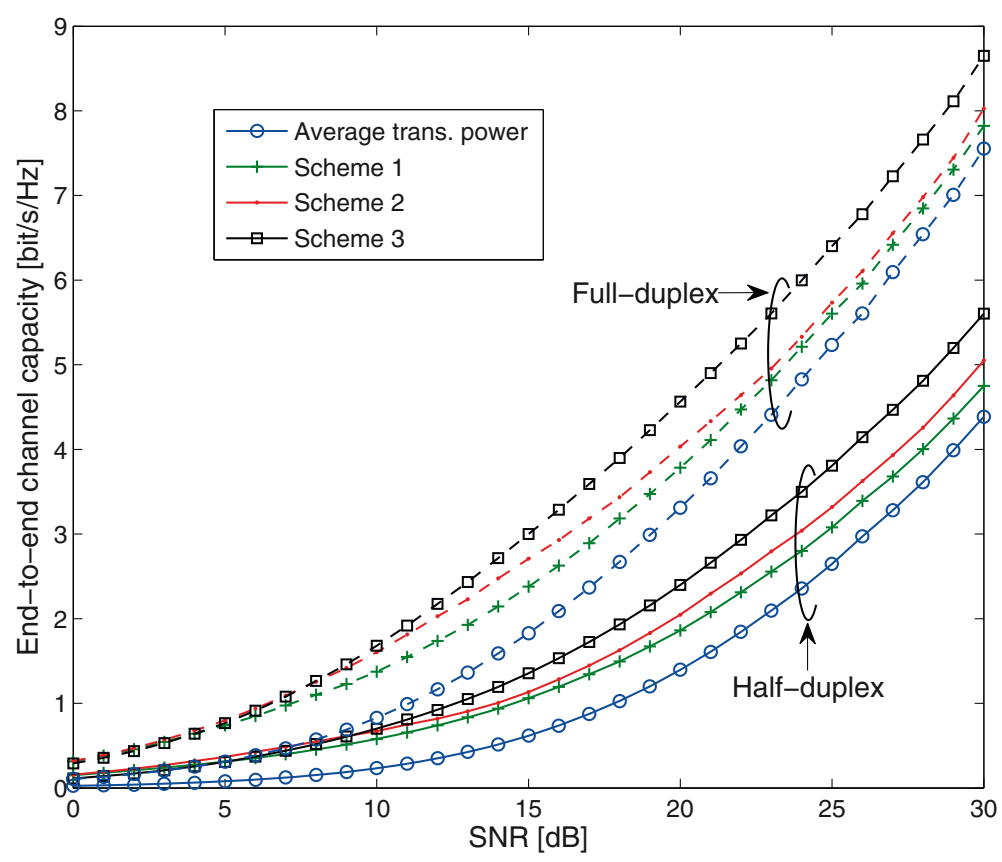

Figure 3 The end-to-end channel capacity of half-duplex and full-duplex AF scheme MMRS with proposed WF method. The end-to-end channel capacity of the average transmit power, and the three WF methods are compared. The end-to-end channel capacity of scheme 3 is the highest, and that of scheme 1 is the lowest. However, the end-to-end channel capacity of the WF method is higher than that of average transmit power. Compared to the half duplex, the end-to-end channel capacity of full duplex is much higher.

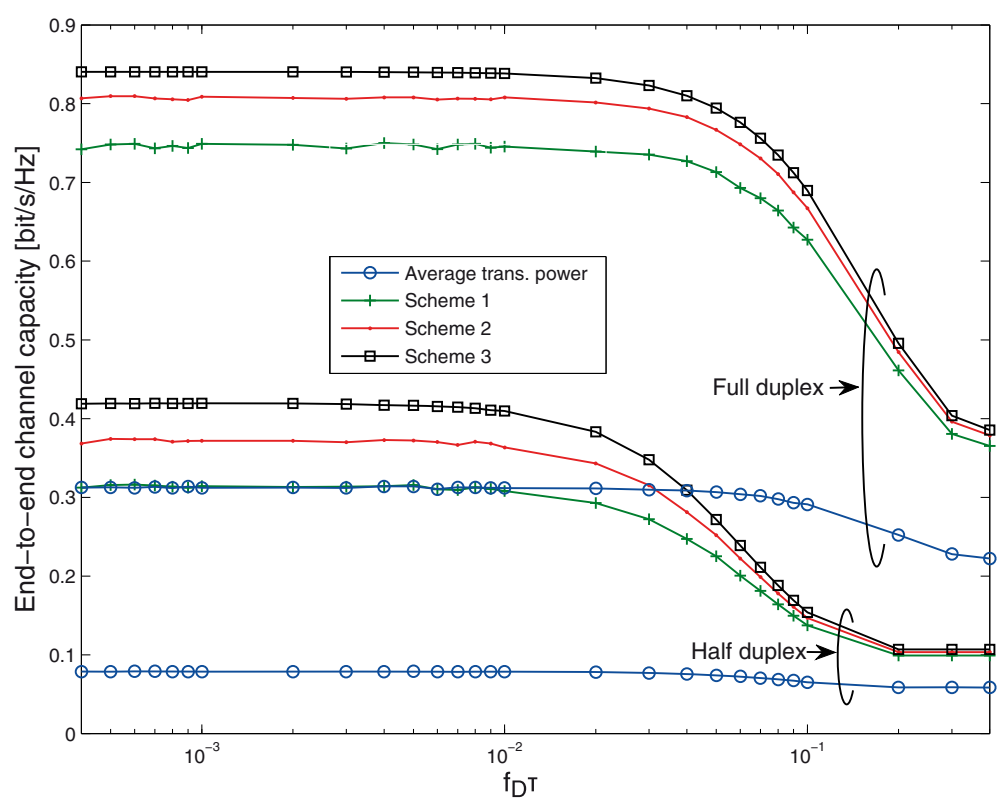

Figure 4 The effect of outdated CSI when SNR $=\mathbf{5} \mathrm{dB}$ and the term $\boldsymbol{f}_{\boldsymbol{D}} \boldsymbol{\tau}$ is changed. The end-to-end channel capacity of outdated CSI system is described. It considerably decreases when the term $f_{D} \tau$ is increased. Compared to that of the half duplex, the end-to-end channel capacity of the full duplex decreases more slowly. 


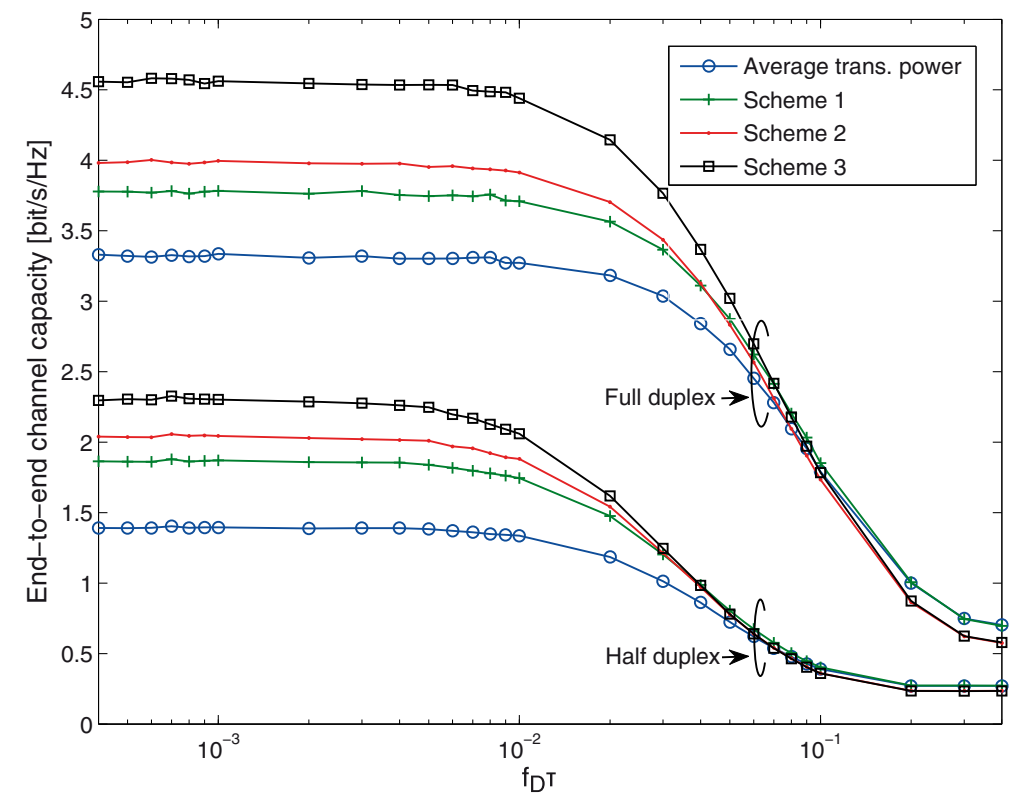

Figure 5 The effect of outdated CSI when SNR $=20 \mathrm{~dB}$ and the term $f_{D} \boldsymbol{\tau}$ is changed. The end-to-end channel capacity of the outdated CSI system is described in the high-SNR region. The end-to-end channel capacity in the high-SNR region decreases more quickly than that in the low-SNR region.

end-to-end channel capacity of WF methods decreases more quickly and be close to that of the average transmit power (Figure 4). The reason is that since the effect of the outdated CSI on co-channel interference is the same in both the WF method and the average transmit power, the reduction of end-to-end channel capacity should be the same. However, the optimization of transmit power of WF methods also be affected by the outdated CSI. The gap between the transmit power and channel models increases when the term $f_{D} \tau$ increases. On the other hand, as shown in Figure 5, the end-to-end channel capacity of WF methods decreases and drops below that of average transmit power. Additionally, compared to $\mathrm{snr}_{i}$ of $5 \mathrm{~dB}$, the end-to-end channel capacity of $\operatorname{snr}_{i}$ of $20 \mathrm{~dB}$ decreases more quickly. It can be explained that since the ratio of desired signal and co-channel interference is fixed as $\frac{1}{\delta(M-1)}$, the power of co-channel interference is high when the power of desired signal is high. It means the signal-to-interference plus noise ratio (SINR) becomes low when the SNR is high. As explained in the previous section, according to the diversity gain of the WF methods, the end-to-end channel capacity as well as the SNR of the WF methods are higher than those of the average transmit power, especially scheme 3 . Therefore, when the term $f_{D} \tau$ increases, the SINRs of schemes 1, 2, and 3 are lower than those of the average transmit power and the SINR of scheme 3 becomes the lowest. It means that the average transmit power is the most robust, and scheme 3 tends to be subjected to the influence of the outdated
CSI. Compared to the half duplex, the end-to-end channel capacity of the full duplex in both the low- and high-SNR region is more robust due to the half of delay time.

The result as explained above can be recognized from Figures 6 and 7 that show the end-to-end channel capacity of half duplex and full duplex, respectively, when the term $f_{D} \tau$ is fixed as 0.001 and 0.05 . When the term $f_{D} \tau$ is small (0.001), the effect of the outdated CSI is not so high; therefore, the end-to-end channel capacity increases when the $\mathrm{snr}_{i}$ increases. However, when the term $f_{D} \tau$ is large (0.05), the effect of the outdated CSI becomes higher; therefore, the end-to-end channel capacity increases slowly, especially in the half-duplex system.

\section{Conclusions}

In this paper, we have analyzed the end-to-end channel capacity of MMRSs according to the proposed WF method. The WF method is divided into three schemes. In scheme 1 , the WF method is applied to each hop. In scheme 2, the transmit power is optimized based on the multiplication of channel models from the Tx to each relay, and in scheme 3 , transmit power of all transmitters is the same and optimized based on multiplication of channel models from the Tx to the Rx. The end-to-end channel capacity of the proposed WF method is higher than that of the average transmit power, and the end-to-end channel capacity of scheme 3 is the highest in the perfect CSI. However, when the imperfect CSI is taken into account, the outdated CSI not only affects the gap between the 


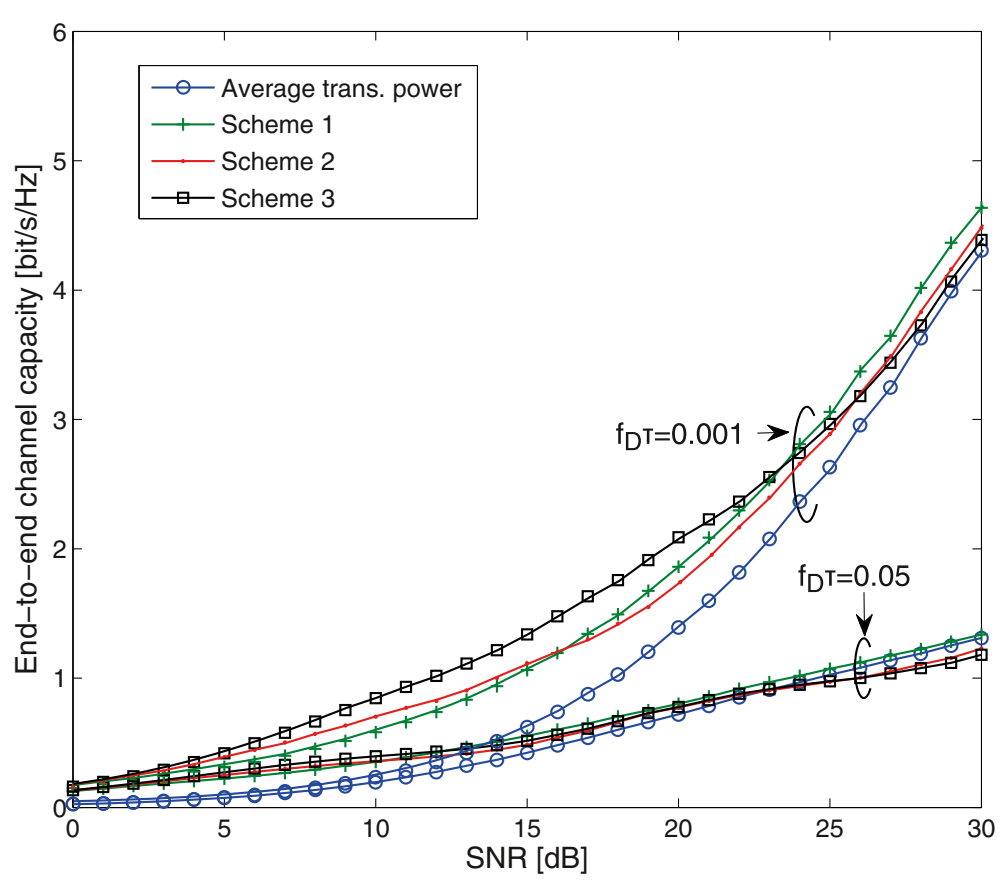

Figure 6 The end-to-end channel capacity of a half-duplex system when $\mathbf{s n r}_{\boldsymbol{i}}$ is changed. The higher the SNR is, the more rapidly the end-to-end channel capacity of the outdated CSI decreases. The average transmit power system is the most robust, and scheme 3 is affected by the outdated CSI much more than the others.

optimized transmit power and channel models but also is effective in the co-channel interference. In the outdated CSI system, the end-to-end channel capacity decreases when the term $f_{D} \tau$ increases, especially in the high-SNR region due to the high power of co-channel interference.
Additionally, the end-to-end channel capacity of the WF methods decreases close to or drops below that of average transmit power. The average transmit power system is the most robust, and scheme 3 is the most sensitive. The half duplex and the full duplex also have been discussed

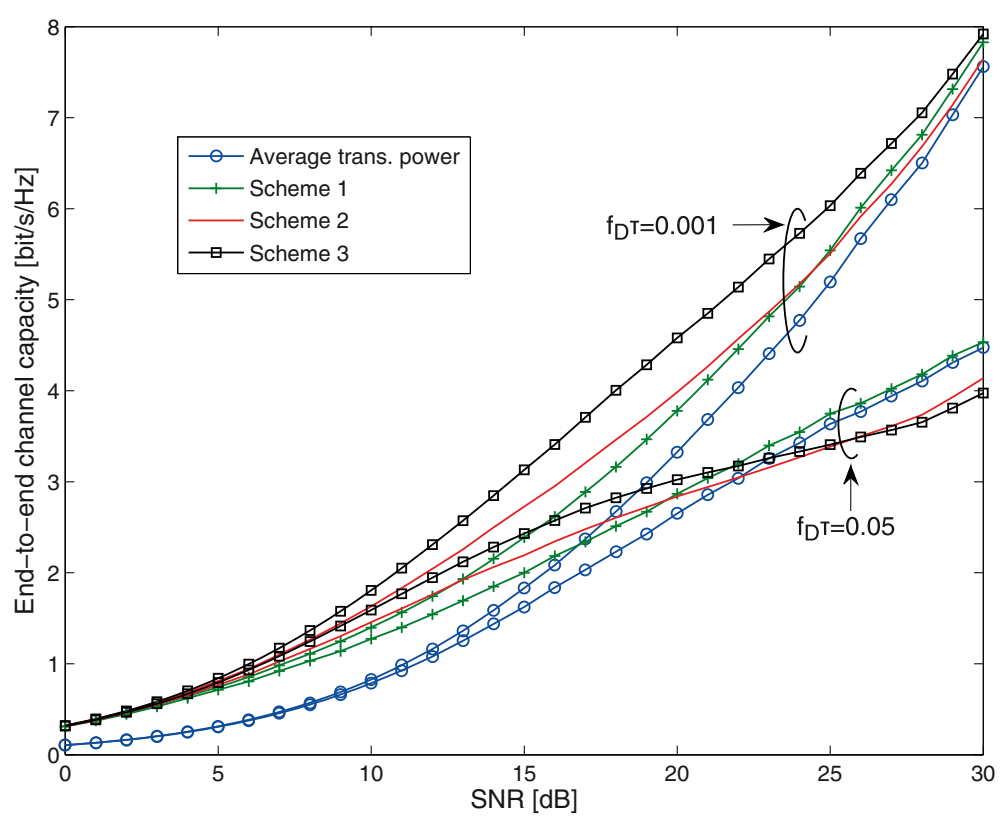

Figure $\mathbf{7}$ The end-to-end channel capacity of a full-duplex system when snr ${ }_{i}$ is changed. Compared to the half duplex, the end-to-end channel capacity of the full-duplex system is more robust due to the half of delay time. 
in this paper. Since the full duplex has a double of allocation time and a half of delay time, the full-duplex system is more robust than the half-duplex system, and the endto-end channel capacity of the full-duplex system is higher than that of the half-duplex system. In this paper, since the full duplex has been taken into consideration, at least four antennas are necessary. The full duplex has more advantages when the number of antennas is greater than or equal to four, whereas the half duplex has more advantages when the number of antennas is less than four. Additionally, the greater the number of antennas is, the higher the end-to-end channel capacity achieves. Moreover, the signal processing of full duplex is considered to be more complicated than that of the half duplex.

Since the distance and the transmit power have been optimized in other literatures, in this paper, the same SNR at all transmitters was assumed. However, the optimization of distance and transmit power based on each WF method should be considered to improve the performance of MMRS. Additionally, the transmission of all transmitters in both the full duplex and the half duplex was assumed to be controlled on MAC layer. However, the detail of control should be discussed. We leave them to the future works.

\section{Competing interests}

The authors declare that they have no competing interests.

Received: 19 December 2013 Accepted: 10 October 2014

Published: 24 October 2014

\section{References}

1. B Wang, J Zhang, A Host-Madsen, On the capacity of MIMO relay channel. IEEE Trans. Inf. Theory. 51, 29-43 (2005)

2. DS Shiu, GJ Foschini, MJ Gans, JM Kahn, Fading correlation and its effect on the capacity of multi-element antenna systems. IEEE Trans. Commun. 48, 502-513 (2000)

3. D Gesbert, H Bolcskei, DA Gore, AJ Paulraj, in Proceedings of GLOBECOM. MIMO wireless channel: capacity and performance prediction, vol. 2 (San Francisco, CA, USA, 27 Nov-01 Dec 2000), pp. 1083-1088

4. Y Liang, W Veeravalli, Gaussian orthogonal relay channels: optimal resource allocation and capacity. IEEE Trans. Inf. Theory. 51, 3284-3289 (2005)

5. B Makki, T Eriksson, On the capacity of Rayleigh-fading correlated spectrum sharing networks. EURASIP J. Wireless Commun. Netw (2011). doi:10.1186/1687-1499-2011-83

6. K Kim, K Ko, J Lee, Adaptive selection of antenna grouping and beamforming for MIMO systems. EURASIP J. Wireless Commun. Netw (2011). doi:10.1186/1687-1499-2011-154

7. Z Shen, RW Heath Jr, JG Andrews, BL Evans, Space-time water-filling for composite MIMO fading channels. EURASIP J. Wireless Commun. Netw (2006). doi:10.1155/WCN/2006/16281

8. Y Lu, W Zhang, in Proceedings of Computing, Communications and IT Applications Conference. Water-filling capacity analysis in large MIMO systems (ComComAp Hong Kong, 1-4 April 2013)

9. K-J Lee, J-S Kim, G Caire, I Lee, Asymptotic ergodic capacity analysis for MIMO amplify-and-forward relay networks. IEEE Trans. Commun. 9, 2712-2717 (2010)

10. S Jin, MR McKay, C Zhong, K-K Wong, Ergodic capacity analysis of amplify-and-forward MIMO dual-hop systems. IEEE Trans. Inf. Theory. 56, 2204-2224 (2010)

11. M Gastpar, M Vetterli, On the capacity of large Gaussian relay networks. IEEE Trans. Inf. Theory. 51, 765-779 (2005)
12. PT Hiep, R Kohno, Optimizing position of repeaters in distributed MIMO repeater system for large capacity. IEICE Trans. Commun. E93-B, 3616-3623 (2010)

13. PT Hiep, O Fumie, K Ryuji, Optimizing distance, transmit power and allocation time for reliable multi-hop relay system. EURASIP J. Wireless Commun. Netw. 2012, 153 (2012)

14. PT Hiep, NH Hoang, S Chika, K Ryuji, End-to-end channel capacity of MAC-PHY cross-layer multiple-hop MIMO relay system with outdated CSI. EURASIP J. Wireless Commun. Netw. 2013, 144 (2013)

15. X Tang, Y Hua, Optimal design of non-regenerative MIMO wireless relays. IEEE Trans. Wireless Commun. 6, 1398-1407 (2007)

16. I Hammerstrom, A Wittneben, Power allocation schemes for amplify-and-forward MIMO-OFDM relay links. IEEE Trans. Wireless Commun. 6, 2798-2802 (2007)

17. S Yang, JC Belfiore, Towards the optimal amplify-and-forward cooperative diversity scheme. IEEE Trans. Inform. Theory. 53, 3114-3126 (2007)

18. B Khoshnevis, W Yu, R Adve, Grassmannian beamforming for MIMO amplify-and-forward relaying. IEEE J. Sel. Areas Commun. 26, 1397-1407 (2008)

19. JB Andersen, Array gain and capacity for known random channels with multiple element arrays at both ends. IEEE J. Sel. Areas Commun. 18, 2172-2178 (2000)

20. Y Karasawa, MIMO propagation channel modeling. IEICE Trans. Commun. E88-B, 1829-1842 (2005)

doi:10.1186/1687-1499-2014-174

Cite this article as: Thanh Hiep and Kohno: Water-filling for full-duplex multiple-hop MIMO relay system. EURASIP Journal on Wireless Communications and Networking 2014 2014:174.

\section{Submit your manuscript to a SpringerOpen ${ }^{\circ}$ journal and benefit from:}

- Convenient online submission

- Rigorous peer review

- Immediate publication on acceptance

- Open access: articles freely available online

- High visibility within the field

Retaining the copyright to your article

Submit your next manuscript at $>$ springeropen.com 